\title{
APLICAÇÃO DO MÉTODO DE MONTE CARLO PARA PREVISÃO DO ÍNDICE DE OVERALL EQUIPMENT EFFECTIVENESS DE UMA MÁQUINA PRODUTORA DE CELULOSE
}

\section{Isabela Gasparino Araújo isabela.ga@alunos.eel.usp.br Universidade de São Paulo, Lorena, São Paulo, Brasil.}

\section{Fabrício Maciel Gomes} fmgomes@usp.br Universidade de São Paulo, Lorena, São Paulo, Brasil.

Félix Monteiro Pereira felixmpereira@usp.br Universidade de São Paulo, Lorena, São Paulo, Brasil.

\section{RESUMO}

A aplicação da simulação nas organizações traz diversas vantagens, desde um entendimento dos seus sistemas e processos, até perspectivas quanto às estratégias e próximos passos, pois permite projetar cenários e desenvolver planos de ação, a baixo custo. Nesse contexto, identificou-se a oportunidade de continuar um estudo referente ao indicador de eficiência global do equipamento, Overall Equipment Effectiveness (OEE), em uma indústria produtora de papel e celulose. Portanto, foram realizadas simulações de Monte Carlo, a partir de dados históricos de natureza quantitativa, referente ao funcionamento de uma máquina - que apresenta OEE médio atual de $65.08 \%$ e capacidade teórica para produzir 624 toneladas de celulose em 24 horas de operação. 0 objetivo foi verificar a viabilidade deste equipamento atingir o nível de $85 \%$ no índice de OEE, considerado de Classe Mundial, e entender quais variáveis e parâmetros mais impactam a eficiência deste indicador, por meio de simulações de Monte Carlo, realizadas no software Crystal Ball. Como resultado, foram realizadas 50.000 iterações e constatou-se que a probabilidade do equipamento atingir um OEE de nível mundial foi de apenas $0.009 \%$. Verificou-se também, a partir do gráfico de sensibilidade, que os parâmetros que mais interferem na eficiência desse índice são o Desempenho (54.7\%) e a Disponibilidade (31.9\%) da máquina. Conclui-se que, a partir da geração de um volume robusto de dados, a simulação permitiu avaliar a interação de diferentes variáveis presentes na linha de produção e seus impactos em indicadores relevantes da empresa, sem a necessidade de realizar qualquer alteração prévia no ambiente de trabalho. Portanto, pode ser aplicada como importante ferramenta para estudos de viabilidade, análise de performance e tomada de decisões nas empresas.

Palavras-chave: Overall Equipment Effectiveness; Celulose; Monte Carlo. 


\section{INTRODUÇÃO}

Nos últimos 30 anos, as estratégias competitivas adotadas pelas indústrias e impulsionadas pela globalização promoveram a criação de redes locais e internacionais de trabalho. Isso levou à terceirização de serviços e à fragmentação da produção para regiões periféricas, sobretudo para o sudeste asiático (Sarti e Hiratuka, 2017). Já com o advento da indústria 4.0, mudanças significativas vêm sendo implantadas nos ambientes fabris, com o uso de tecnologias digitais para coleta e análise de dados em tempo real, que proporcionam informações relevantes sobre o processo, fundamentais para o direcionamento das empresas (Frank et al., 2019).

Nesse contexto, para aproveitar de maneira eficiente os recursos disponíveis, com foco na geração de produtos de alta qualidade, com o mínimo de retrabalho e resíduos, são realizadas avaliações de desempenho da produção, que é o primeiro passo para aplicação de melhorias e ações corretivas e/ou preventivas no ambiente de trabalho (Roda e Macchi, 2019). Isso se torna ainda mais relevante para empresas que trabalham com produtividade inferior a $50 \%$, que atingem esse percentual por não possuírem estratégias e um planejamento mínimo da produção (Pinto et al., 2018).

Uma das abordagens está relacionada ao estudo da capacidade produtiva nas indústrias, realizado sobretudo a partir da quantidade total de bens produzidos versus o tempo necessário para execução (Tang, 2019). Essas ações visam identificar perdas ocultas relacionadas ao sistema de produção, assim como desenvolver indicadores e planos que possam controlar seus efeitos ao longo do tempo (Roda e Macchi, 2019). Isso porque com bons resultados de produtividade é possível aumentar a satisfação dos clientes, reduzir o tempo e o custo para criar, produzir e entregar serviços de qualidade (Plinere e Aleksejeva, 2019)

Uma pesquisa realizada pela Confederação Nacional da Indústria (CNI), que abrange 12 estados brasileiros, com atividade industrial que corresponde à $93,9 \%$ do Produto Interno Bruto (PIB) industrial, mostra que o rendimento médio real no setor diminuiu $0,7 \%$ entre janeiro e fevereiro de 2019 (CNI, 2019).

Já a capacidade instalada das indústrias em fevereiro de 2019 foi de $78 \%$, em que houve queda de 0,3 pontos percentuais, quando comparado com o mesmo período em 2018 (CNI, 2019). Com relação aos investimentos realizados pelas indústrias em 2017, 64\% foram direcionados para compra de equipamentos e máquinas, seguido de aquisição de tecnologias com $24 \%$, que englobam desde automações até soluções digitais (CNI, 2018).

Esses dados, referente ao cenário brasileiro, auxiliam no entendimento de que ainda existe espaço para melhorias e que, portanto, faz-se necessário aprofundar os estudos sobre produtividade nas empresas para se antecipar ao mercado, que se torna cada vez mais dinâmico. Nessa conjuntura, pode-se destacar a utilização dos métodos de modelagem como ferramenta de apoio para projeção de cenários e desenvolvimento de estudos diversos de viabilidade, executados a baixo custo como meio para conhecimento de sistemas complexos que dependem da análise de diferentes variáveis (Silva et al., 2018).

Portanto, este trabalho visa abordar o uso da simulação de Monte Carlo como ferramenta complementar à tomada de decisão e como um meio para análise de riscos, visto que permite levantar a situação atual do objeto em estudo, com informações e dados que servem como subsídios para realizar inferências sobre o futuro, a partir da determinação de parâmetros específicos e suas respectivas distribuições de probabilidade.

\section{FUNDAMENTAÇÃO TEÓRICA}

\subsection{Overall Equipment Effectiveness (OEE)}

O OEE está inserido no contexto da Manutenção Produtiva Total (TPM), sendo empregado como um indicador para medir a eficiência global de equipamentos, que utiliza para a base de cálculo informações coletadas na linha de produção (Roda e Macchi, 2019). Foi introduzido nas indústrias japonesas por Seiichi Nakajima, por volta de 1970. Desde então, tornou-se uma ferramenta quantitativa de medição da produtividade, fundamental nas indústrias (Foulloy et al., 2019).

Como diferencial, esse indicador envolve as áreas que atuam direta e indiretamente na produção, portanto, diversos setores da empresa conseguem contribuir para melhorar a eficiência dos seus equipamentos, com o cumprimento de metas individuais que impactam no desempenho do OEE (Almeida e Fabro, 2019). O OEE é uma métrica que relaciona a utilização de instalações, tempo e recursos, além de indicar possíveis gaps entre o desempenho real e o ideal. Para isso, são avaliados três parâmetros, conforme descrito por Sitompul e Rinawati (2019): disponibilidade, desempenho e qualidade.

\section{Disponibilidade}

A disponibilidade refere-se aos tempos de funcionamento e de inatividade da máquina. Entre os fatores que influenciam esse indicador, destacam-se:

a) Tempo de carregamento: tempo total de disponibilidade de uso do equipamento para produzir, em um dia. 
b) Tempo planejado de produção: tempo necessário para atender a demanda programada no dia;

c) Tempo de inatividade planejado: tempo já predefinido em que a máquina não vai operar, como nos intervalos de refeição dos colaboradores e manutenção preventiva;

d) Tempo de inatividade não planejado: tempo de inatividade não especificado, como falta de energia e defeito da máquina.

Sendo assim, a taxa de disponibilidade pode ser expressa conforme a equação 1 .

$$
\text { Disponibilidade }=\frac{T P P-T I N P}{T D C-T I P} \times 100
$$

Onde:

TPP = Tempo planejado de produção (horas)

TINP = Tempo de inatividade não planejado (horas)

TDC $=$ Tempo de carregamento (horas)

TIP = Tempo de inatividade planejado (horas)

\section{Desempenho}

O indicador de desempenho compara a quantidade produzida com a demanda previamente programada. A taxa de desempenho é calculada conforme a equação 2 e os tópicos abaixo:

a) Tempo de operação: tempo real necessário para atender à demanda da produção;

b) Quantidade produzida: volume total de material produzido pela máquina;

c) Tempo de ciclo ideal: tempo necessário para fabricar uma unidade de produto.

$$
\text { Desempenho }=\frac{Q P-T C I}{T O} \times 100
$$

Onde:

$\mathrm{QP}=$ Quantidade produzida (unidades)

$\mathrm{TCl}=$ Tempo de ciclo ideal (horas)

TO = Tempo de operação (horas)

\section{Qualidade}

O indicador de qualidade trata da quantidade total de bens produzidos, sem defeitos. Para tanto, dois fatores são utilizados como referência:

a) Quantidade processada: volume de todos os produtos fabricados em um dia;

b) Falhas: quantidade de produtos defeituosos produzidos em um dia.

A taxa de qualidade é calculada de acordo com a equação 3

$$
\text { Qualidade }=\frac{Q P R-F}{Q P R} \times 100
$$

Onde:

QPR = Quantidade processada (unidades)

$\mathrm{F}=$ Falhas (unidades)

Todos esses parâmetros que são calculados em percentuais apontam para diferentes pontos do processo, que podem ser melhorados individualmente. A equação 4 relaciona esses indicadores e nos fornece a função de eficiência global do equipamento.

OEE $=$ Disponibilidade $\times$ Desempenho $\times$ Qualidade

O OEE que resulta em $100 \%$ equivale a um sistema produtivo perfeito, em um tempo ótimo, sem inatividade e geração de peças defeituosas. Já o percentual de $85 \%$ é considerado de classe mundial, sendo uma meta alcançada por empresas de alto desempenho. O OEE em $60 \%$ indica que há espaço para melhorias, e o de $40 \%$ (considerado bastante baixo), é típico em empresas recém-criadas que ainda estão ajustando e entendendo seus processos (Plinere e Aleksejeva, 2019).

De acordo com Sitompul e Rinawati (2019), para um OEE de classe mundial, que corresponde ao percentual de $85 \%$, o valor mínimo individual de cada parâmetro deve ser de $90 \%$ para disponibilidade, $95 \%$ para desempenho e $99,9 \%$ para qualidade.

Para sistemas produtivos que não utilizam métricas temporais, como os processos de galvanização, pode-se calcular - OEE a partir de uma equação genérica, representada na equação 5 (Almeida e Fabro, 2019). 


$$
\mathrm{m}_{\text {global }}=\frac{\text { Produtividade real }}{\text { Produção teórica }}
$$

Esses indicadores podem ser comprometidos quando: peças defeituosas são geradas; há a necessidade de retrabaIho; ocorrem paradas por causa da configuração e manutenção da máquina; ocorre transição entre operações ou perdas de rendimento desde a inicialização do equipamento até sua estabilização (Sousa et al., 2018). Entretanto, de maneira geral, por meio de manutenções periódicas, por exemplo, espera-se que a confiabilidade e a capacidade das máquinas sejam melhoradas (Figueiredo e Oliveira, 2019).

\section{Simulação}

A simulação é uma ferramenta da pesquisa operacional e, na engenharia, é comumente utilizada para investigar características de sistemas, em que os resultados podem ser aplicados para entendimento de um outro processo, que seja similar (Silva et al., 2018). Auxilia também no planejamento e direcionamento das organizações, permitindo valorar ganhos e perdas com base nas alternativas projetadas, sem impacto no sistema real, considerando a variabilidade e as incertezas do ambiente (Lucena et al., 2019).

A simulação é realizada com o uso de um algoritmo, conhecido como solucionador, que interpreta o comportamento de um sistema real e produz inferências sobre o seu estado futuro (Thule et al., 2019). Sendo assim, permite estimar cenários de um modelo que pode ser experimentado sob diferentes condições. $\mathrm{O}$ cálculo é realizado a partir de uma aproximação entre as variáveis de entrada, que devem corresponder ao estado atual do objeto em estudo, e os dados de saída criados pela simulação (Thule et al., 2019).

Para Silva et al. (2018), a simulação pode ramificar-se em dois tipos. Primeiro, em modelos contínuos, que analisa sistemas cujas características se alteram ao longo do tempo, em que são aplicadas equações diferenciais para análise das variáveis. Segundo, em modelos discretos, que descrevem a evolução de um sistema como reação a uma série de eventos, como os problemas de filas de espera, em que são realizadas projeções baseadas em novos tempos de espera e composição de filas. Thule et al. (2019) ainda destacam o modelo híbrido, que combina características dos modelos contínuos e discretos.

Lucena et al. (2019) apresenta quatro procedimentos sequenciais para desenvolvimento de uma simulação. Primeiro, deve-se planejar, ou seja, entender o problema e listar os recursos necessários para coleta de dados e execução dos experimentos. Nessa etapa, é importante realizar um levantamento bibliográfico para contextualização do estudo e escolha das melhores técnicas e ferramentas a serem utilizadas na pesquisa. Em seguida, é necessário criar um modelo matemático a partir do mapeamento dos fluxos atrelados ao sistema em estudo, diagramas complementares, coleta e iteração dos dados.

Na etapa de verificação e validação, o modelo gerado é testado e analisado se suas características correspondem ao do sistema real. Além disso, também é realizado um estudo sobre a sua precisão, o quanto os valores dos dados gerados são próximos entre si (Thule et al., 2019). Por fim, são realizados experimentos, com a projeção do modelo em diferentes ambientes e condições operacionais. $O$ pesquisador analisará os resultados simulados, atrelando informações importantes para a tomada de decisão, como desempenho do modelo, custos e disposição de recursos (Lucena et al., 2019).

No ambiente industrial, a simulação como ferramenta de suporte à tomada de decisão do negócio é, em partes, limitada, porque não se ajusta em tempo real a ambientes complexos e em constante mudança (como variabilidade da demanda e introdução de novos produtos e/ou equipamentos) em razão da dificuldade em reconfigurar manualmente os parâmetros do modelo (Goodall et al., 2019).

Nesse contexto, a partir dos anos 1970, vêm sendo realizados estudos e técnicas computacionais para acelerar a execução de softwares de simulação, com o uso, por exemplo, de múltiplos processadores em paralelo conectados em rede. Essa abordagem é investigada pela Ciência da Computação e é chamada de Distributed Simulation.

Atualmente, como alternativa aos pesquisadores que não possuem infraestrutura em Tecnologia da Informação (TI) para simular grandes modelos, os serviços em nuvem oferecem espaço de armazenamento para criação de aplicativo Big Data e Analytics de alto desempenho a baixo custo (Taylor, 2019).

Além disso, com a produção de elementos cibernéticos aplicados a ambientes de simulação, múltiplos componentes são acoplados entre si para obter o comportamento total de vários sistemas analisados simultaneamente. Para tanto, as saídas de um simulador são conectadas às entradas de outros, o que gera um amplo mapeamento do sistema e dos ambientes adjacentes (Thule et al., 2019). Sendo assim, os estudos, teorias e tecnologias, aplicados no contexto da simulação, fornecem cada vez mais insumos para explorar e trabalhar as limitações atreladas ao método, assim como perspectivas quanto a sua expansão e aplicabilidade para todas as áreas do conhecimento. 


\subsection{SIMULAÇÃO DE MONTE CARLO}

A simulação de Monte Carlo é conhecida há séculos, entretanto, o primeiro artigo referente ao método foi publicado em 1949 por Metropolis e Ulam (Yoriyaz, 2009). Foi durante a Segunda Guerra Mundial que ganhou notoriedade ao ser aplicado pelos cientistas John Von Neumann e Stanislaw Ulam em simulações para determinar a probabilidade de ocorrer fissões nucleares, a partir dos coeficientes de difusão de nêutrons, no desenvolvimento de uma bomba atômica (Silva et al., 2019). Já a nomenclatura deriva dos populares jogos de azar, realizados em cassinos na cidade de Monte Carlo, em Mônaco (Silva et al., 2018).

Monte Carlo é definido como um método estocástico - conjunto de variáveis aleatórias parametrizadas, utilizadas para estudar um sistema ao longo do tempo (Giraldo et al., 2018) - que gera uma quantidade massiva de dados para cada variável do sistema que está sendo analisado. Os resultados não serão os mesmos em cada simulação, mas tendem a convergir para valores próximos (Amorim et al., 2018).

Essa abordagem requer o emprego de softwares e pode ser aplicada para funções lineares e não-lineares, visto que não depende da natureza do modelo (Martin et al., 2018). Quanto maior o número de simulações, maior será a precisão do método. No entanto, maior também será o tempo e a capacidade computacional exigida para armazenamento dos dados gerados (Nascimento et al., 2018).

Esta simulação tem como objetivo reproduzir um sistema real por um determinado período de tempo, que fornece dados artificiais utilizados para fazer inferências relacionadas ao processo em estudo. Além disso, simula qualquer sistema que dependa de fatores aleatórios (Silva et al., 2017). Com os resultados é possível realizar cálculos de natureza descritiva, tais como média, desvio-padrão, mediana, valores mínimo e máximo (Silva et al., 2018).

Para Osaki et al. (2019), existem duas limitações significativas atreladas ao método de Monte Carlo que devem ser consideradas ao aplicá-lo em um estudo. $O$ primeiro deles seria a dificuldade em determinar, algebricamente, a distribuição de probabilidade das variáveis analisadas, quando não há conhecimento prévio suficiente sobre a base de dados. O segundo problema está em identificar se de fato há uma relação de interdependência entre os números aleatórios gerados pelo modelo, já considerando que eles seguem uma distribuição predeterminada.

Monte Carlo é um método que pode ser aplicado em áreas diversas, desde gestão de projetos a análises físico-químicas. Por exemplo, dentro do contexto esportivo, em que os resultados dos jogos são bastante incertos e depen- dem de variáveis dinâmicas. Nascimento et al. (2018) aplicaram a simulação de Monte Carlo, via cadeias de Markov, para prever a classificação dos times de futebol no campeonato brasileiro de 2017, utilizando como base a média de gols dos jogos passados de cada rodada. Foram calculadas as probabilidades de os times vencerem, empatarem ou perderem.

Visando atender uma necessidade no campo da Engenharia da Qualidade, Otsuka e Nagata (2018) estudaram a aplicação de Monte Carlo para determinar os índices de tolerância de peças ainda na fase de projeto, com o objetivo de diminuir a variação do processo e o consequente aumento nos custos de fabricação. Isso porque as dimensões reais das peças não correspondem às especificações definidas durante o projeto, devido a procedimentos de usinagem, o que afeta o desempenho final, que depende de um bom encaixe de todas as peças do produto. $O$ método foi utilizado para projetar os valores limites de tolerância de cada peça, a partir da média e do desvio-padrão, de forma que após o procedimento de montagem, o desempenho do produto final não fosse comprometido.

Já no cenário da mobilidade urbana, Goes et al. (2018) verificaram os riscos atrelados à ocorrência de acidentes em uma rede viária na cidade de Fortaleza, Ceará, utilizando como variáveis o volume médio diário de veículos e a velocidade média no trecho, que possui tráfego intenso de cargas. Para a aplicação de Monte Carlo, foram realizadas 1.000 iterações e concluiu-se que a quantidade de simulações geradas foi eficaz para avaliar o impacto de cada parâmetro no estudo.

\section{MÉTODO}

Para previsão dos índices de OEE foi utilizada a simulação de Monte Carlo, a partir de uma pesquisa de natureza descritiva, que tem como finalidade analisar a relação entre as variáveis em estudo, sendo necessário descrevê-las, classificá-las e interpretá-las.

Foi empregada a abordagem quantitativa, que aplica técnicas estatísticas tanto na coleta, como no tratamento dos dados, mensuráveis, de maneira objetiva. Esse método visa descobrir o relacionamento entre variáveis e para representação dessas informações, normalmente são utilizados gráficos e tabelas. Por fim, é possível generalizar os resultados obtidos a partir de um estudo amostral para toda a população da pesquisa (Fernandes et al., 2018).

Os procedimentos adotados para aplicação da simulação de Monte Carlo acompanharam os tópicos listados abaixo, conforme adaptado de Pérez et al. (2019): 
a) Definir as variáveis de entrada do sistema analisado;

b) Modelar essas variáveis;

c) Identificar as distribuições probabilísticas de cada uma delas;

d) Configurar e executar a simulação de Monte Carlo em um software;

e) Analisar os resultados gerados.

Em complemento, utilizou-se o software Crystal Ball para realização das simulações.

\subsection{CRYSTAL BALL}

Para o tratamento, simulação e análise dos dados foi utilizada a ferramenta Crystal Ball, versão 11.1.4716.0, criada pela empresa de origem americana Oracle Corporation. Funciona como uma extensão das planilhas MS Excel e trata-se de um software também utilizado para aplicação de Monte Carlo, desenvolvido para projetos de simulação, modelagem e otimização, em que a cada iteração os dados gerados são armazenados. Para o resultado final é possível realizar cálculos estatísticos de natureza descritiva, o que permite ao pesquisador a projeção de diferentes cenários também em estudos futuros (Amorim et al., 2018).

Emprega-se o método meta-heurístico, que explora uma série de valores possíveis para as variáveis do problema em análise, na busca por uma solução ótima, em vez de diferenciação matemática, base de cálculo de softwares clássicos de otimização (Mantzaras e Voudrias, 2017).

Para utilização do Crystal Ball como ferramenta coadjuvante nas simulações de Monte Carlo, deve-se primeiro definir as distribuições de probabilidade das variáveis de entrada e posteriormente realizar algumas configurações no software, que impactam nos resultados esperados, como quantidade de iterações desejadas, velocidade da simulação e intervalo de confiança. Após a simulação, é gerado um relatório, que inclui gráficos de

frequência, de sensibilidade e de correlação, tabelas com estatísticas descritivas e informações sobre as distribuições mais prováveis para a variável resposta, entre outros dados.

\subsection{OBJETO DE ESTUDO}

Como base de dados para o desenvolvimento deste trabalho, foi utilizado um estudo de caso dirigido por Sitompul e Rinawati (2019) em uma indústria de celulose, onde foi calculado o índice de OEE para a máquina Digester, que tem a capacidade teórica de produzir 624 toneladas de celulose em um tempo de operação de 24 horas. Diante disso, observou-se a oportunidade de expandir o estudo de eficiência global do equipamento em

uma simulação, como mecanismo de previsão para tomada de decisão antecipada, considerando os dados históricos de operação da máquina e referenciais teóricos do indicador levantados na literatura.

A cadeia produtiva de papel e celulose envolve pelo menos cinco grandes etapas: a produção de madeira (predominantemente o eucalipto), de energia, celulose, papel e sua posterior reciclagem (Oliveira et al., 2018). Apesar da expansão dos recursos tecnológicos, a produção de papel vem crescendo e seu consumo mundial já ultrapassou 400 milhões de toneladas, com média global de $55 \mathrm{~kg}$ por pessoa (Sanquetta et al., 2019). No Brasil, dos 7,84 milhões de hectares de árvores plantadas para fins industriais, 35\% são destinados para produção de papel e celulose (IBA, 2019).

\subsection{BASE DE DADOS}

Para levantamento dos dados, coletados em agoto de 2016, Sitompul e Rinawati (2019) realizaram pesquisas na linha de produção, por meio de observação direta e entrevistas com os operadores da fábrica, obtendo informações referentes às horas de trabalho, quantidade de material produzido, tempo de inatividade e resíduos gerados na máquina Digester. A partir dessas informações, foi possível calcular os indicadores de disponibilidade, desempenho e qualidade do equipamento, assim como o índice de OEE, apresentados naTabela 1.

As variáveis de entrada apresentadas são dados quantitativos e de natureza contínua. Duas delas possuem valores fixos, capacidade de produção e tempo de operação teórico, respectivamente 624 toneladas e 24 horas. As demais variáveis (tempo de operação real,

volume de falhas e produção total) se comportam conforme as condições do ambiente e do próprio equipamento.

Conforme mapeamento realizado previamente no referido estudo, as informações foram extraídas de duas linhas de trabalho na fábrica, que impactam diretamente no funcionamento da máquina Digester: a seção de energia, que faz gestão das fontes de energia utilizadas nos processos, e a seção de linhas de fibras, que realiza desde o corte da madeira até o empacotamento das folhas de celulose (Sitompul e Rinawati, 2019).

Apesar da máquina funcionar 24 horas por dia, houve 
Tabela 1. Base de dados da máquina Digester

\begin{tabular}{cccccccc}
\hline Medição & Tempo Esperado (h) & Produção (ton) & Falhas (ton) & Disponibilidade & Desempenho & Qualidade & OEE (\%) \\
\hline 1 & 22,00 & 560,39 & 70,97 & 0,917 & 0,898 & 0,873 & 71,90 \\
2 & 21,85 & 517,53 & 24,292 & 0,910 & 0,829 & 0,953 & 71,96 \\
3 & 21,55 & 539,8 & 36,202 & 0,898 & 0,865 & 0,933 & 72,47 \\
4 & 21,00 & 548,93 & 70,681 & 0,875 & 0,880 & 0,871 & 67,06 \\
5 & 21,40 & 548,53 & 72,622 & 0,892 & 0,879 & 0,868 & 68,01 \\
6 & 19,75 & 535,22 & 70,796 & 0,823 & 0,858 & 0,868 & 61,25 \\
7 & 18,70 & 506,16 & 70,738 & 0,779 & 0,811 & 0,860 & 54,37 \\
8 & 19,80 & 524,66 & 70,721 & 0,825 & 0,841 & 0,865 & 60,02 \\
9 & 14,85 & 362,69 & 70,683 & 0,619 & 0,581 & 0,805 & 28,96 \\
10 & 16,90 & 415,58 & 70,607 & 0,704 & 0,666 & 0,830 & 38,93 \\
11 & 21,35 & 571,23 & 72,444 & 0,890 & 0,915 & 0,873 & 71,11 \\
12 & 21,85 & 551,26 & 72,629 & 0,910 & 0,883 & 0,868 & 69,83 \\
13 & 21,45 & 598,18 & 72,575 & 0,894 & 0,959 & 0,879 & 75,28 \\
14 & 21,55 & 577,18 & 48,546 & 0,898 & 0,925 & 0,916 & 76,07 \\
15 & 21,95 & 527,11 & 0,000 & 0,915 & 0,845 & 1,000 & 77,26 \\
16 & 18,25 & 428,53 & 0,000 & 0,760 & 0,687 & 1,000 & 52,22 \\
17 & 21,65 & 556,51 & 52,018 & 0,902 & 0,892 & 0,907 & 72,93 \\
18 & 21,30 & 527,47 & 48,337 & 0,888 & 0,845 & 0,908 & 68,15 \\
19 & 20,95 & 505,54 & 34,480 & 0,873 & 0,810 & 0,932 & 65,90 \\
20 & 21,95 & 546,98 & 65,765 & 0,915 & 0,877 & 0,880 & 70,53 \\
21 & 21,15 & 549,99 & 73,075 & 0,881 & 0,881 & 0,867 & 67,35 \\
22 & 18,50 & 492,55 & 11,995 & 0,771 & 0,789 & 0,976 & 59,36 \\
23 & 14,55 & 347,13 & 0,000 & 0,606 & 0,556 & 1,000 & 33,73 \\
24 & 21,35 & 551,21 & 0,000 & 0,890 & 0,883 & 1,000 & 78,58 \\
25 & 21,65 & 528,06 & 41,383 & 0,902 & 0,846 & 0,922 & 70,36 \\
26 & 21,55 & 572,72 & 41,692 & 0,898 & 0,918 & 0,927 & 76,41 \\
27 & 21,25 & 549,17 & 41,483 & 0,885 & 0,880 & 0,924 & 72,04 \\
28 & 20,75 & 570,09 & 41,445 & 0,865 & 0,914 & 0,927 & 73,25 \\
29 & 20,55 & 570,16 & 41,476 & 0,856 & 0,914 & 0,927 & 72,55 \\
30 & 21,95 & 561,96 & 41,408 & 0,915 & 0,901 & 0,926 & 76,30 \\
31 & 16,00 & 432,28 & 25,878 & 0,667 & 0,693 & 0,940 & 43,42 \\
\hline & & & 509 & &
\end{tabular}

Fonte: Adaptado de Sitompul e Rinawati (2019).

paradas diversas ao longo das medições, por ser necessário realizar ajustes no motor da caldeira quando o desempenho da energia diminuía. A produção também era interrompida para realização de reparos e alimentação da máquina com matéria prima.

Como análise inicial, pode-se constatar que os indicadores médios de eficiência global da máquina Digester são de $84.58 \%$ para disponibilidade, $83.62 \%$ para desempenho, $91.05 \%$ para qualidade e $65.08 \%$ para OEE. São percentuais baixos e ainda distantes do que hoje é classificado como de classe mundial (Plinere e Aleksejeva, 2019).

\subsection{CONFIGURAÇÃO DOS DADOS DA SIMULAÇÃO NO CRYSTAL BALL}

\subsubsection{Disponibilidade, desempenho e qualidade}

Como ponto de partida, foram realizadas simulações para verificar quais as probabilidades dos percentuais de disponibilidade, desempenho e qualidade da máquina Digester atingir os níveis considerados de classe mundial que, conforme Sitompul e Rinawati (2019), são, respectivamente, de $90 \%, 95 \%$ e $99,9 \%$. Para tanto, os dados históricos do equipamento foram utilizados para definição das distribuições estatísticas das variáveis de entrada. 
De acordo com a Tabela 2, foi adotada a distribuição triangular, de natureza contínua, por melhor se ajustar à base de dados.

Tabela 2. Distribuição estatística das variáveis de entrada.

\begin{tabular}{cccc}
\hline Variável & Distribuição & Parâmetros & $\begin{array}{c}\text { Valores dos } \\
\text { parâmetros }\end{array}$ \\
\hline $\begin{array}{c}\text { Produção Real } \\
\text { (ton) }\end{array}$ & \multirow{2}{*}{ Triangular } & $\begin{array}{c}\text { Mínimo } \\
\text { Moda }\end{array}$ & 347,13 \\
& & Máximo & 521,77 \\
Falhas (ton) & \multirow{2}{*}{ Triangular } & Mínimo & 0 \\
& & Moda & 46,93 \\
\multirow{2}{*}{ Tempo de } & \multirow{2}{*}{ Triangular } & Mínimo & 73,08 \\
Produção Real (h) & Moda & 14,55 \\
& & Máximo & 20,30 \\
& & & 22,00 \\
\hline
\end{tabular}

As unidades de medidas das variáveis de entrada foram toneladas e horas e, para a variável de saída, percentual. Para cada rodada, foram realizadas 50.000 iterações no software Crystal Ball, em conjunto com o MS Excel, com intervalo de confiança de $95 \%$. Ainda no software foram configurados as distribuições e os parâmetros para cada variável de entrada, conforme definidos anteriormente na Tabela 2.

\subsubsection{Overall Equipment Effectiveness}

Com o objetivo de determinar qual dos parâmetros gera maior impacto no resultado do OEE, foi realizada uma segunda etapa. A partir das informações da Tabela 1, foram verificadas as distribuições estatísticas das variáveis de disponibilidade, desempenho e qualidade, conforme exposto na Tabela 3. Verificou-se que a distribuição triangular melhor se ajustou aos dados de disponibilidade e desempenho. Já os dados de distribuição lognormal aos dados de qualidade.

Tabela 3. Distribuição estatística dos parâmetros do OEE.

\begin{tabular}{cccc}
\hline Variável & Distribuição & Parâmetros & $\begin{array}{c}\text { Valores dos } \\
\text { parâmetros }\end{array}$ \\
\hline \multirow{2}{*}{ Disponibilidade } & Triangular & Mínimo & 0,6063 \\
& & Moda & 0,8458 \\
& & Máximo & 0,9167 \\
Desempenho & \multirow{2}{*}{ Triangular } & Mínimo & 0,5563 \\
& & Moda & 0,8362 \\
& & Máximo & 0,9586 \\
Qualidade & \multirow{2}{*}{ Lognormal } & Local & 0,4210 \\
& & Média & 0,9106 \\
& & Desvio Pa- & 0,0508 \\
\hline
\end{tabular}

Da mesma forma, foram realizadas 50.000 iterações no software Crystal Ball, com o auxílio das planilhas MS Excel, definido intervalo de confiança de $95 \%$ e configurados as distribuições e parâmetros das variáveis de entrada apresentadas anteriormente na Tabela 3.

\section{RESULTADOS E DISCUSSÕES}

A princípio, os parâmetros de disponibilidade, desempenho e qualidade foram simulados individualmente para verificar a probabilidade de atingirem os níveis de classe mundial, assim como para identificar quais das variáveis de entrada impactam de maneira significativa cada um desses indicadores. Em um segundo momento, foi estimada a probabilidade de o OEE do equipamento ser igual ou superior a $85 \%$, percentual de referência a nível mundial, e quais dos parâmetros mais afetam o resultado final desse indicador na máquina Digester.

\subsection{Disponibilidade}

A simulação do parâmetro de disponibilidade durou 89,84 segundos e, conforme Tabela 4, é possível observar a estatística descritiva dos dados gerados na previsão. Nota-se um desvio padrão baixo $(0,07)$, um bom indicador de que os valores simulados apresentaram baixa variabilidade (coeficiente de variação de $8,41 \%$ ). O valor máximo foi de 0,92 , um pouco além do valor teórico de 0,90, considerado de classe mundial, porém a amplitude dos dados da previsão foi significativa, de 0,31 .

Tabela 4. Estatística descritiva da simulação: Disponibilidade

\begin{tabular}{cc}
\hline Estatística & Valor da Previsão \\
\hline Média & 0,79 \\
Mediana & 0,80 \\
Moda & 0 \\
Desvio Padrão & 0,07 \\
Variância & 0 \\
Obliquidade & $-0,4442$ \\
Curtose & 2,40 \\
Coeficiente de Variação & 0,0841 \\
Valor Máximo & 0,92 \\
Valor Mínimo & 0,61 \\
\hline
\end{tabular}

Verifica-se ainda que a distribuição não é simétrica, pois os valores de média, mediana e moda diferem entre si. Para entender melhor o formato da distribuição, pode-se analisar a curtose, que indica a dispersão dos dados no gráfico de frequência. Para este caso a curtose foi positiva (2.40), ou seja, caldas com maior concentração de valores, portanto, uma distribuição mais achatada. Para fins de comparação, uma distribuição normal apresenta curtose igual a zero.

Com relação à probabilidade de o percentual de disponibilidade ser igual ou maior do que $90 \%$, conforme Figura 1, foi de apenas $1.77 \%$. 


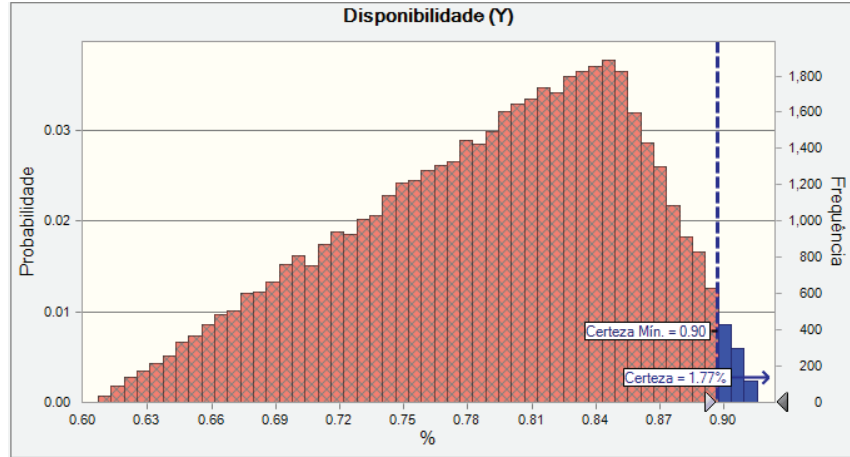

Figura 1. Gráfico de frequência da simulação de Disponibilidade

Sobre a variável de maior impacto, pode-se observar no gráfico de sensibilidade, conforme Figura 2 que, para este estudo, o tempo de operação (100\%) é o único fator que influencia o parâmetro de disponibilidade. Portanto, cabe avaliar a necessidade de investimentos para implantação de melhorias que objetivam a redução do lead time, revisões nos procedimentos que envolvam a utilização do equipamento e alterações no layout da fábrica, que possam auxiliar para um melhor aproveitamento do tempo disponível para utilização da máquina.

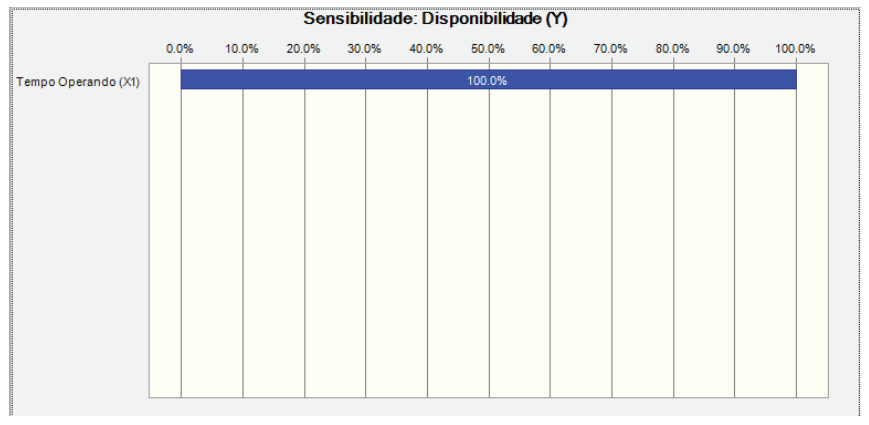

Figura 2. Gráfico de sensibilidade da simulação de Disponibilidade

Além disso, para aumentar a confiabilidade do equipamento, deve-se elaborar uma agenda para definir a periodicidade em que serão realizadas manutenções preventivas e preditivas na máquina, visto que atualmente ocorrem paradas não planejadas em razão das quebras. Um cronograma de manutenção também pode auxiliar na dificuldade que o equipamento possui de estabilizar sua operação no momento em que é inicializado, como apontado por Sitompul e Rinawati (2019).

A distribuição que melhor se adequou à variável de resposta foi a triangular (máximo: 0.92, mínimo: 0.61, mais provável: 0.85).

\subsection{Desempenho}

Esta simulação durou 97.59 segundos e observa-se que a média dos dados simulados (0.78) foi ligeiramente inferior à média dos dados históricos (0.83), conforme Tabela 5 . O desvio padrão e a variância deste parâmetro também foram baixos, de 0.08 e 0.01 respectivamente. Esse fatos indicam uma baixa dispersão dos dados, referente ao valor esperado (coeficiente de variação de $10.72 \%$ ). Já a amplitude foi significativa, de 0.40 , visto que o valor mínimo foi de apenas 0.56 . A obliquidade, que faz referência à assimetria da distribuição, foi negativa. Isso indica um volume maior de dados na região esquerda do gráfico, onde estão os valores abaixo da média.

Tabela 5. Estatística descritiva da simulação: Desempenho

\begin{tabular}{cc}
\hline Estatística & Valor da Previsão \\
\hline Média & 0,78 \\
Mediana & 0,79 \\
Moda & 0 \\
Desvio Padrão & 0,08 \\
Variância & 0,01 \\
Obliquidade & $-0,3491$ \\
Curtose & 2,41 \\
Coeficiente de Variação & 0,1072 \\
Valor Máximo & 0,96 \\
Valor Mínimo & 0,56 \\
\hline
\end{tabular}

Já a probabilidade do percentual de desempenho ser igual ou maior a 95\%, considerado de classe mundial, foi de apenas $0.088 \%$, como mostra a Figura 3.

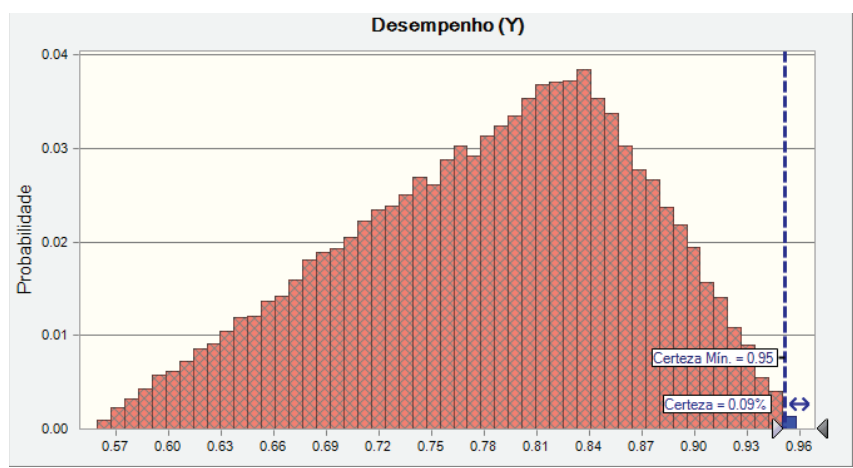

Figura 3. Gráfico de frequência da simulação de Desempenho.

O gráfico de sensibilidade, apresentado na Figura 4, nos indica que a única variável que causa impacto no parâmetro de desempenho, neste estudo, é a produção real (100\%). Portanto, a quantidade de celulose processada pelo equipamento está diretamente relacionada ao percentual de desempenho, que conforme dados históricos apresentados na Tabela 1, em nenhum momento o equipamento foi capaz de produzir o volume ótimo esperado, de 624 toneladas por dia. 


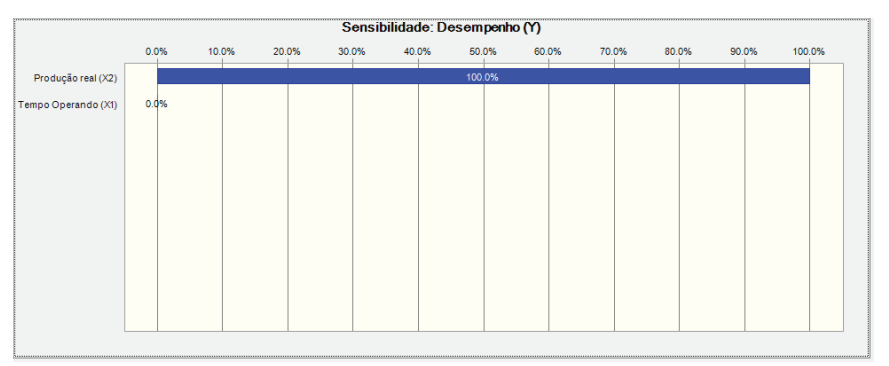

Figura 4. Gráfico de sensibilidade da simulação de Desempenho

Este parâmetro pode ser melhorado, de maneira geral, com a diminuição de retrabalho e produções defeituosas. Além disso, deve ser avaliada a velocidade em que o equipamento executa os seus comandos, por exemplo, por meio de uma cronoanálise, que ainda vai evidenciar os principais gargalos do processo, a partir do mapeamento de cada etapa do processo. $O$ indicador também se relaciona aos momentos em que a produção foi interrompida, devido à execução de reparos na máquina.

A distribuição que melhor se adequou à variável de resposta foi a triangular (máximo: 0.96 , mínimo: 0.56 , mais provável: 0.83$)$.

\subsection{Qualidade}

A simulação deste indicador durou 83.65 segundos e, conforme Tabela 6, gerou estatísticas descritivas que se destacaram. Por exemplo, entre as simulações dos três paramentos: menores desvio padrão (0.03), coeficiente de variação (0.036) e maior valor máximo (1.00). Além disso, os dados gerados na simulação obtiveram média de 0.92 , praticamente igual à média da qualidade dos dados históricos do equipamento, que foi de 0.9106 . Já a obliquidade deste indicador foi positiva, o que nos mostra que houve concentração de dados na região direita da calda no gráfico de frequência, relacionada aos valores acima da média.

Tabela 6. Estatística descritiva da simulação: Qualidade

\begin{tabular}{cc}
\hline Estatística & Valor da Previsão \\
\hline Média & 0,92 \\
Mediana & 0,92 \\
Moda & 0 \\
Desvio Padrão & 0,03 \\
Variância & 0 \\
Obliquidade & 0,0342 \\
Curtose & 2,62 \\
Coeficiente de Variação & 0,0360 \\
Valor Máximo & 1,00 \\
Valor Mínimo & 0,80 \\
\hline
\end{tabular}

Sobre a probabilidade do equipamento conseguir atingir um percentual igual ou superior a $99.9 \%$ no indicador de qualidade, a simulação nos forneceu que essa chance foi de apenas $0.196 \%$, conforme Figura 5.

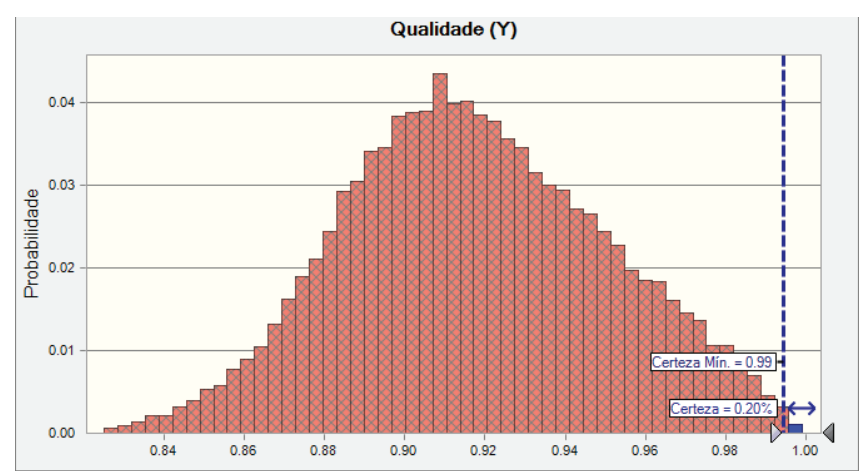

Figura 5. Gráfico de frequência da simulação de Qualidade.

A variável que causou maior efeito neste parâmetro foi a quantidade de falhas $(92,6 \%)$, conforme gráfico de sensibilidade demonstrado na Figura 6. A princípio, pode-se avaliar se os picos de falha possuem correlação com determinados turnos (pois a fábrica funciona durante 24 horas por dia) e com os operadores, nos momentos em que ocorre inicialização ou encerramento da máquina, sazonalidade em épocas do ano (devido à temperatura/ umidade/pressão do ambiente que podem impactar no funcionamento da máquina), entre outros fatores ambientais relevantes.

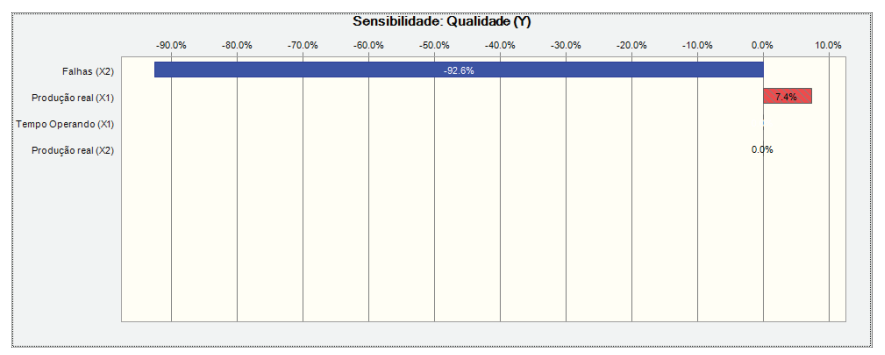

Figura 6. Gráfico de sensibilidade da simulação de Desempenho

Em complemento, a utilização de ferramentas da qualidade, como o diagrama de Ishikawa, auxilia no mapeamento da causa raiz dos problemas que afetam este indicador, que incluem os pontos anteriormente citados, como também a avaliação sobre quais etapas do processo geram maior quantidade de falhas, a partir da análise das entradas e saídas, dos procedimentos, recursos e ferramentas utilizados.

A distribuição que melhor se adequou à variável de resposta foi a beta (máximo: 1.04, mínimo: 0.80, alfa: 6.06234, beta: 6.48598$)$. 


\subsection{Overall Equipment Effectiveness}

Para o indicador de OEE o tempo de simulação foi de 70.34 segundos e, conforme Tabela 7 com as estatísticas descritivas da base de dados da previsão, a média foi de apenas 0.56, inferior à média histórica do OEE da máquina Digester, de 0.6508. Este indicador apresentou a maior amplitude (0.60) entre as simulações realizadas neste estudo, o que evidencia a variabilidade do processo (maior coeficiente de variação entre as simulações,

0.1476). Além disso, a distribuição apresentou uma ligeira concentração de dados na região direita, onde estão os valores superiores à média, visto que sua obliquidade foi baixa e positiva (0.0546).

Tabela 7. Estatística descritiva da simulação: OEE

\begin{tabular}{cc}
\hline Estatística & Valor da Previsão \\
\hline Média & 0,56 \\
Mediana & 0,56 \\
Moda & 0 \\
Desvio Padrão & 0,08 \\
Variância & 0,01 \\
Obliquidade & 0,0546 \\
Curtose & 2,67 \\
Coeficiente de Variação & 0,1476 \\
Valor Máximo & 0,90 \\
Valor Mínimo & 0,30 \\
\hline
\end{tabular}

Já o gráfico de frequência, Figura 7, que demonstra a probabilidade do indicador de alcançar percentual igual ou superior a $85 \%$, índice de OEE de classe mundial, para este equipamento foi de $0.009 \%$.

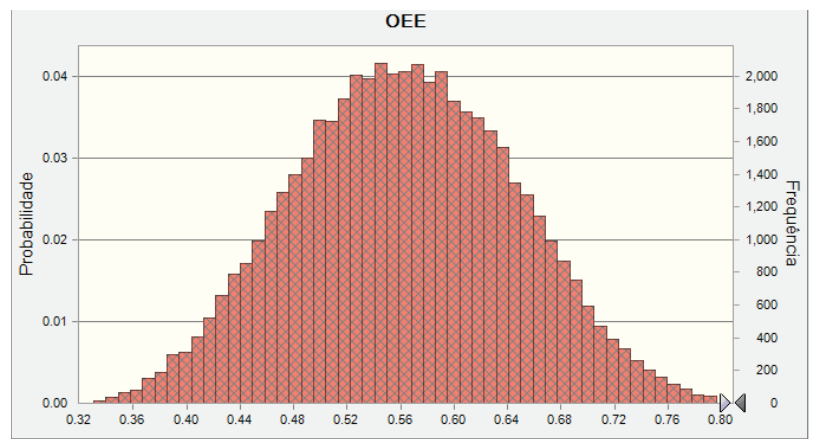

Figura 7. Gráfico de frequência da simulação do OEE.

O gráfico de sensibilidade, Figura 8, nos retornou quais parâmetros mais impactam o OEE. Para a máquina Digester, o desempenho $(54.7 \%)$ e a disponibilidade (31.9\%) juntos somam $87 \%$. Este percentual retorna informações importantes sobre em quais etapas do processo a gerência da empresa deve priorizar as ações para melhoria do indicador e, consequentemente, da sua produtividade. Almeida e Fabro (2019) alertam que as análises e decisões que interferem no OEE precisam ser realizadas com cuidado, visto a importância de um equipamento atingir níveis de excelência, pois é um fator que compromete a sustentabilidade de uma empresa dentro do mercado.

Já a distribuição que melhor se adequou à variável de resposta foi a beta (máximo: 0.91, mínimo: 0.25, alfa: 6.84837, beta: 7.60708).

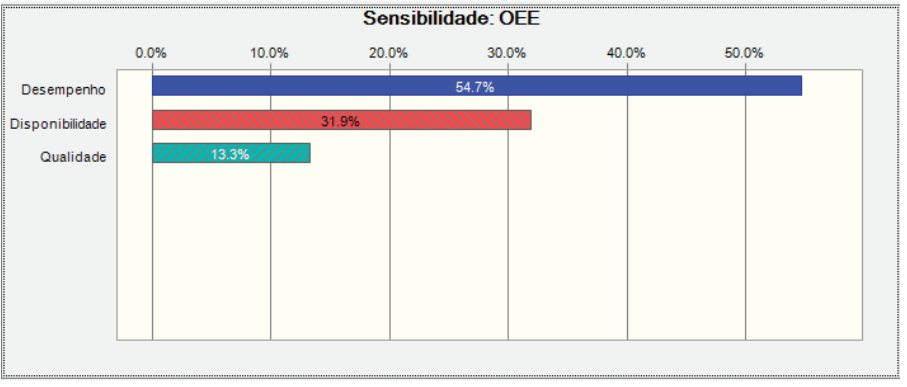

Figura 8. Gráfico de sensibilidade da simulação do OEE

Por fim, a partir dos resultados discutidos neste capítulo, é possível afirmar que a simulação fornece maiores insumos para a empresa, no que tange ao entendimento dos seus processos e gargalos, a partir de uma perspectiva mais ampla. Como as variáveis históricas são limitadas a um período específico e ao tamanho das medições, a simulação de Monte Carlo conseguiu gerar uma quantidade de informações bastante relevante (50.000 iterações), baseado no comportamento real do equipamento, que auxilia o pesquisador na investigação de correlações que talvez não fossem possíveis com um volume reduzido de dados.

Para este estudo, verificou-se que as variáveis tempo de operação e produção foram as mais relevantes, e que devem ser priorizadas pela empresa em uma tomada de decisão voltada para investimentos em melhorias, por exemplo. São alterações diretamente ligadas aos três parâmetros, porém destacam-se os indicadores de desempenho e de disponibilidade como os fatores de maior impacto no resultado final do OEE da máquina Digester.

Não coincidentemente, referenciando-se aos dados históricos do equipamento, o desempenho e a disponibilidade estão distantes do que hoje é classificado como de classe mundial e na simulação apresentaram maior coeficiente de variação (0.1072 e 0.0841), ou seja, maior variabilidade nos dados gerados, quando comparados com o parâmetro de qualidade, uma avaliação mediana quando se trata de planejamento e controle da produção.

Além disso, destaca-se que a máquina foi capaz de alcançar o percentual máximo no indicador de qualidade (100\%), na simulação e no funcionamento real do equipamento. Como consequência, direciona a empresa no entendimento 
de que é interessante também investir no aperfeiçoamento deste indicador, com intervenções talvez mais acessíveis no dia a dia, pois este parâmetro apresentou melhores estatísticas e resultados que os demais.

Já sobre o uso do Crystal Ball como software para simulação dos dados, destaca-se a simplicidade para utilização e interpretação das informações geradas (desde tabelas estatísticas à gráficos de frequência e sensibilidade), o que facilita sua aplicação para pesquisadores/analistas de empresas que não possuem conhecimento prévio sobre a ferramenta e a simulação de Monte Carlo.

\section{CONCLUSÃO}

A simulação de Monte Carlo é uma opção acessível e descomplicada para ser aplicada em diferentes situações, dada a sua universalidade. No cenário aqui analisado, destacou-se a velocidade em que ocorreram as simulações, com tempo médio de 85 segundos. Além disso, o principal objetivo deste estudo foi alcançado, pois foi possível identificar os principais fatores e parâmetros que impactam significativamente no índice de OEE da máquina Digester.

Conclui-se também que as probabilidades do equipamento atingir os níveis mundiais de referência para desempenho (0.088\%), disponibilidade (1.77\%), dualidade (0.196\%) e OEE (0.009\%) foram bastante reduzidas. Entretanto, este cenário apenas reforça sobre a necessidade de se analisar a linha de produção a partir de uma perspectiva que englobe do micro ao macro, pois tanto os parâmetros ligados diretamente com a eficiência do equipamento, como o ambiente da empresa podem impactar no resultado.

\section{REFERÊNCIAS}

Almeida, B.G., Fabro, E. 2019. Indústria 4.0 como ferramenta na engenharia de manutenção com base na metodologia TPM. Scientia cum Industria 7, 23-39.

Amorim, F.R., Abreu, P.H.C, Patino, M.T.O., Terra, L. A. A. 2018. Análise dos Riscos em Projetos: Uma Aplicação do Método de Monte Carlo em uma Empresa do Setor Moveleiro. Future Studies Research Journal: Trends \& Strategies, 10, 332-357.

CNI. 2018. Investimentos na indústria: 2017/2018. n. 1. Recuperado em 14 de abril de 2019. http://www. portaldaindustria.com.br/estatisticas/investimentos-naindustria/

CNI. 2019. Indicadores industriais: fevereiro 2019. n. 2. Recuperado em 14 de abril de 2019. http://www. portaldaindustria.com.br/estatisticas/indicadoresindustriais/
Fernandes, A.M., Bruchêz, A., d'Ávila, A.A.F., Castilhos, N.C., e Olea, P.M. 2018. Metodologia de pesquisa de dissertações sobre inovação: Análise bibliométrica. Desafio Online 6, 141-159.

Figueiredo, O. C., Oliveira, U. R. 2019. Resultados Empíricos do Mapeamento do Fluxo de Valor em Uma Empresa Automotiva. Revista Gestão Industrial 15, 39-63.

Foulloy, L., Clivillé, V., Berrah, L. 2019. A fuzzy temporal approach to the Overall Equipment Effectiveness measurement. Computers \& Industrial Engineering 127, 103-115.

Frank, A.G., Dalenogare, L.S., Ayala, N.F. 2019. Industry 4.0 technologies: Implementation patterns in manufacturing companies. International Journal of Production Economics 210, 15-26.

Giraldo, C.A.S., Mendoza, J.S.D., Jálabe, A.M. 2018. Impacto de los costos de calidad en la ejecución de los proyectos de construcción en Colombia. Revista Escuela de Administración de Negocios, 33-54.

Goes, G.V., Márcio de Almeida, D.A., Bertoncini, B.V., Goes, G. V. 2018. Vulnerabilidade da rede viária urbana: avaliação considerando risco e emissão de gases de efeito estufa. Revista Brasileira de Gestão Urbana, 10, 159-172.

Goodall, P., Sharpe, R., West, A. 2019. A data-driven simulation to support remanufacturing operations. Computers in Industry, 105, 48-60.

IBA. Panorama brasileiro. 2019. Recuperado em 14 de abril de 2019 https://iba.org/dados-estatisticos

Lucena, A.J.F., Silva, L.A., Araújo, P.P.P., Carneiro, T.D.C. 2019. Modelagem e simulação na gestão de recursos humanos: um estudo de caso aplicado as equipes de patrulhamento da polícia militar na cidade de Caicó-RN. Revista Livre de Sustentabilidade e Empreendedorismo 4, 5-27.

Mantzaras, G., Voudrias, E.A. 2017. An optimization model for collection, haul, transfer, treatment and disposal of infectious medical waste: Application to a Greek region. Waste Management, 69, 518-534.

Martin, A.J., Karthikeyan, S., Karthikeyan, P.R.K.M.V. 2018. Manufacturing tolerance design using monte carlo method. Taga Journal 14, 2214-2221.

Nascimento, L., Santana, J.M.M., Macedo, J.B., Moura, M.C. 2018. Modelo de previsão da classificação do campeonato brasileiro 2017 utilizando simulação Monte Carlo via cadeias de Markov. Anais do Encontro Nacional de Engenharia de Produção, Maceió. AL, Brasil, 38.

Oliveira, A.B., Pereira, J.M., Nascimento, A.A. 2018. Cadeia produtiva de papel e celulose e transformações recentes no sudoeste maranhense. InterEspaço: Revista de Geografia e Interdisciplinaridade 4, 135-154. 
Osaki, M., Alves, L.R.A., Lima, F.F., Ribeiro, R.G., Barros, G.S.A.D.C. 2019. Risks associated with a double-cropping production system-a case study in southern Brazil. Scientia Agricola 76, 130-138.

Otsuka, A., \& Nagata, F. 2018. Quality design method using process capability index based on Monte-Carlo method and real-coded genetic algorithm. International Journal of Production Economics 204, 358-364.

Pérez, P., Aguado, S., Albajez, J. A., \& Santolaria, J. 2019. Influence of laser tracker noise on the uncertainty of machine tool volumetric verification using the Monte Carlo method. Measurement 133, 81-90.

Pinto, L. G., Castro, R. G., Tanajura, L. L. C., Santos, F. K. G., \& de Lisboa Sucupira, C. R. 2018. Conceitos e Fatores Determinantes para o Alcance da Produtividade. Ideias e Inovação-Lato Sensu 4, 123-130.

Plinere, D., \& Aleksejeva, L. 2019. Production scheduling in agent-based supply chain for manufacturing efficiency improvement. Procedia Computer Science 149, 36-43.

Roda, I., \& Macchi, M. 2019. Factory-level performance evaluation of buffered multi-state production systems. Journal of Manufacturing Systems 50, 226-235.

Sanquetta, C. R., de Oliveira, T. W. G., Dalla Corte, A. P., Sanquetta, M. N. I., \& Maas, G. C. B. 2019. Análise da produção, importação, exportação e consumo aparente de papel no brasil entre 1961 e 2016. BIOFIX Scientific Journal 4, 110-115.

Sarti, F., \& Hiratuka, C. 2017 Desempenho recente da indústria brasileira no contexto de mudanças estruturais domésticas e globais. Texto para discussão (UNICAMP), Campinas, 290, 1-20.
Silva, C. V., Souza, A. B., Sales, H. L., \& Penha, R. S. 2019. Aplicação do modelo monte Carlo na avaliação da empresa Ambev com custo de capital impreciso. Revista Eniac Pesquisa 8, 153-175.

Silva, E. F., Rodrigues, L. S., \& Damasceno, L. F. F. 2018. Previsão de demanda por meio do método de simulação de monte carlo em uma loja de conveniência. South American Development Society Journal 4, 244.

Silva, V. A. G., Almeida, T. S., Ribeiro, R. J. A. 2017. A utilização de gráfico de controle e método de Monte Carlo em um estudo de caso de desperdício em uma empresa fabricante de tintas. Anais do Encontro Nacional de Engenharia de Produção, Joinville, SC, Brasil, 37.

Sitompul, B. G., \& Rinawati, D. I. 2019. Analisis overall equipment effectiveness (oee) pada mesin digester dan pendekatan 5 whys untuk perbaikan pada pt toba pulp lestari, Tbk.(Studi Kasus: PT TOBA PULP LESTARI, Tbk.). Industrial Engineering Online Journal 8.

Sousa, E., Silva, F. J. G., Ferreira, L. P., Pereira, M. T., Gouveia, R., \& Silva, R. P. 2018. Applying SMED methodology in cork stoppers production. Procedia Manufacturing, 17, 611-622.

Tang, H. 2019. A new method of bottleneck analysis for manufacturing systems. Manufacturing letters 19, 21-24.

Taylor, S. J. 2019. Distributed simulation: state-of-the-art and potential for operational research. European Journal of Operational Research, 273, 1-19.

Thule, C., Lausdahl, K., Gomes, C., Meisl, G., \& Larsen, P. G. 2019. Maestro: The INTO-CPS co-simulation framework. Simulation Modelling Practice and Theory, 92, 45-61.

Yoriyaz, H. 2009. Método de Monte Carlo: princípios e aplicações em Física Médica. Revista Brasileira de Física Médica, 3, 141-149.

Recebido: 05 fev. 2020

Aprovado: 06 mar. 2020

DOI: $10.20985 / 1980-5160.2020 . v 15 n 1.1608$

Como citar: Araujo, I.G., Gomes, F.M., Pereira, F.M. (2020), Aplicação do método de Monte Carlo para previsão do índice de Overall Equipment Effectiveness de uma máquina produtora de celulose, Revista S\&G 15, No. 1, $25-37$. https://revistasg.emnuvens.com.br/sg/article/view/1608 